IBIMA Publishing

Journal of Research \& Developments in Chemistry

http://www.ibimapublishing.com/journals/CHEM/chem.html

Vol. 2015 (2015), Article ID 157781, 5 pages

DOI: $10.5171 / 2015.157781$

Research Article

\title{
The Study on the Photocatalytic Degradation of Nicotine
}

\author{
Ewa Adamek $^{1}$, Maciej Goniewicz ${ }^{2}$, Wojciech Baran $^{3}$ and Andrzej Sobczak ${ }^{4}$ \\ ${ }^{1,3}$ Medical University of Silesia, Department of General and Analytical Chemistry, Sosnowiec, Poland \\ ${ }^{2}$ Oncology Department of Health Behavior Division of Cancer Prevention and Population Sciences, \\ Roswell Park Cancer Institute Elm \& Carlton Streets, Buffalo, New York, USA \\ ${ }^{4}$ Institute of Occupational Medicine and Environmental Health, Kościelna, Sosnowiec, Poland
}

Correspondence should be addressed to: Ewa Adamek; ewa.adamek@wp.pl

Received date: 30 April 2014; Accepted date: 12 June 2014; Published date: 17 April 2015

Academic Editor: Hildegard M. Schuller

Copyright (C) 2015. Ewa Adamek, Maciej Goniewicz, Wojciech Baran and Andrzej Sobczak. Distributed under Creative Commons CC-BY 4.0

\begin{abstract}
Nicotine belongs to a group of compounds being specific toxic anthropogenic contaminants. Highly carcinogenic nitrozamines are one of the possible products of photocatalytic oxidation of nicotine. The main objective of the present study was to investigate the photocatalytic degradation of nicotine and the intentification of 4-(methylnitrosamino)-1(3-pyridyl)-1-butanone and N-nitroso-nornicotine among their degradation products.The experiment was carried out in an aqueous medium, in the presence of $\mathrm{TiO}_{2}$ suspension $(0.4$ $\mathrm{g} / \mathrm{l})$. Samples containing nicotine $(0.1 \mathrm{mmol} / \mathrm{l})$ or nicotine and $\mathrm{NO}_{2}-$ ions were irradiated with UVa radiation $\left(\lambda_{\max }=366 \mathrm{~nm}\right)$. Photocatalytic degradation products were identified by using LC-MS/MS method. It was found that during UVa-irradiation of samples with $\mathrm{TiO}_{2}$ the decomposition of nicotine was observed. It can be noted that under investigated conditions the formation of nitrosamines was not observed in any samples.
\end{abstract}

Keywords: nicotine, nitrosamine, degradation, photocatalysis, $\mathrm{TiO}_{2}$

\section{Introduction}

Nicotine belongs to the specific, toxic pollutants of the air. Its presence is unavoidable in air purification systems in rooms where smoking is permitted. Tar, accompanying nicotine causes rapid filling the pores used in such sorbents or filters. Therefore, the use of degradation processes of organic pollutants in such systems seems to be right and proper. According to Bezak-Mazur and Adamczyk
(2010), the simultaneous use of sorbents and advanced oxidation processes (AOPs) increases the total efficiency of degradation process and significantly extends the life of the sorbents. One of the new methods which can be used for destructive removal of contaminants from water and air is the photocatalytic degradation process. It bases mainly on the oxidation of pollutants using hydroxyl radicals ( $\mathrm{HO}^{\circ}$ ) generated by photoexcitation of photocatalyst particles

Cite this Article as: Ewa Adamek, Maciej Goniewicz, Wojciech Baran and Andrzej Sobczak (2015), " The Study on the Photocatalytic Degradation of Nicotine", Journal of Research \& Developments in Chemistry, Vol. 2015 (2015), Article ID 157781, DOI: 10.5171/2015.157781 
(Carp et al., 2004, Fujishima et al., 2008, Ohtani, 2010).

These radicals can be used to break down contaminants that are both hazardous and resistant to biodegradation and turn them into less toxic substances (Baran et al.
2006). However, the oxygen compounds of nitrogen $\left(\mathrm{NO}_{\mathrm{x}}\right)$ may be also generated during the photocatalytic process in the presence of air. As a result, they can be converted to strongly carcinogenic nitrosamines in the presence of nicotine (Fig. 1) (Hecht et al., 1978, Hecht et al., 1993, Sleiman et al., 2010).

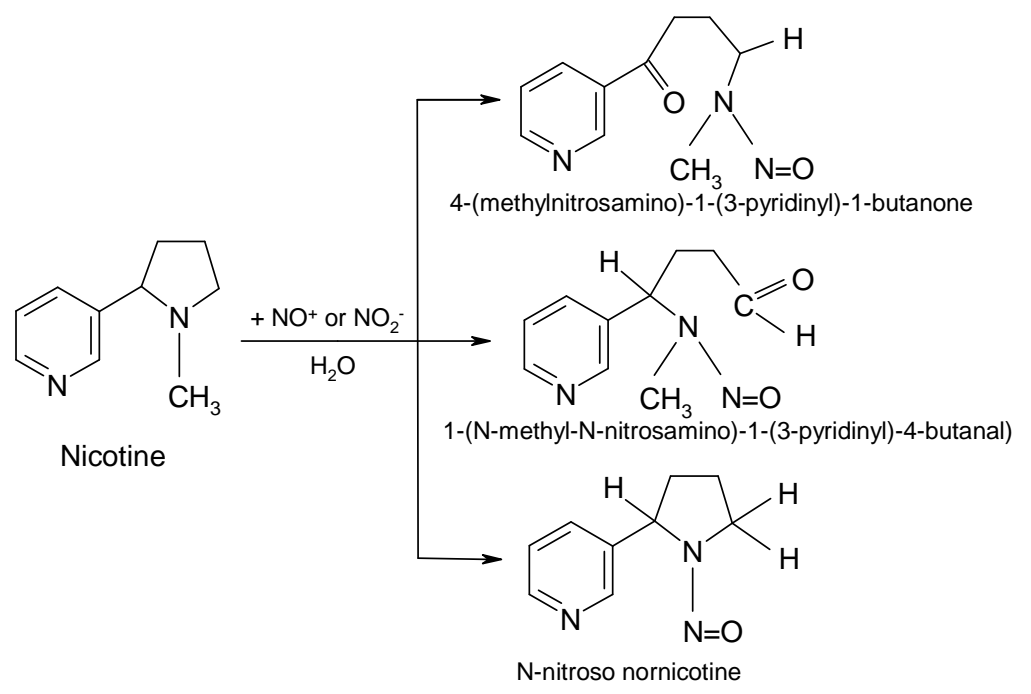

Figure 1: Nitrosamines formed during the conversion of nicotine with oxygen compounds of nitrogen $\left(\mathrm{NO}_{\mathrm{x}}\right)$

The possible formation of nitrosamines during the photocatalytic process can undermine the desirability of its application in air purification systems. The aim of our work was to study the photocatalytic degradation of nicotine and to try to identify 4-(methylnitrosamino)-1(3-pyridyl)-1-butanone and N-nitrosonornicotine among its photodegradation products.

\section{Materials and methods}

The working solutions were prepared in the form of aqueous solutions of nicotine $(0.1 \mathrm{mmol} / \mathrm{l})$ to which $0.4 \mathrm{~g} / \mathrm{l}$ of solid $\mathrm{TiO}_{2}$ (anatase powder, Riedel de Haën) was added. Additionally, the solid $\mathrm{NaNO}_{2}$ (Sigma Aldrich; assay $\geq 99 \%$ ) was added to one sample $(0.1 \mathrm{mmol} / \mathrm{l})$ The photocatalytic process was carried out in open, glass and flat vessels. The prepared mixtures were irradiated by fluorescent lamps (Philips Actinic BL TL40W/10) with a wavelength maximum $(\lambda)$ at $366 \mathrm{~nm}$.
Samples were magnetically stirred and had a free contact with atmospheric air. At predetermined sampling time intervals (before- and during irradiation), the aliquots (between 1 and $2 \mathrm{ml}$ ) of mixtures were collected and centrifuged (20 min, 4000 RPM). All samples were analyzed by liquid chromatography (Agilent 1200 HPLC) equipped with HSF5 column $(4.5 \times 150 \mathrm{~mm}$, $5 \mu \mathrm{m}$ particle size, Supelco) and coupled with quadrupole mass spectrometer (TSQ Quantum Ultra triple quadrupole massspectrometer stage, Thermo-Finnigan, San Jose, CA). The elution was performed at 0.9 $\mathrm{ml} / \mathrm{min}$ flow rate using the gradient mode elution. The mobile phase was a mixture of methanol (25-100\%) and an aqueous solution of ammonium formate (10 mmol/l). The MS/MS detector was operated in the system for automatic detection of the investigated compounds. The following mass-to-charge $(\mathrm{m} / \mathrm{z})$ ratios were used for detection: 178 and 148 for 4(methylnitrosamino)-1-(3-pyridyl)-1butanone and 208 and 122 for N-nitroso- 
nornicotine, at collision energy 8 and $12 \mathrm{eV}$, respectively.

\section{Results and Discussion}

The photocatalytic degradation process of nicotine was carried out in aqueous solution rather than in a gas. Such a procedure should reduce the volatility of formed intermediates and increase the possibility of their retention in the reaction medium. It was found that during UV-a irradiation the concentration of nicotine was reduced. This fact confirms its degradation under investigated conditions. The dynamics of the process is shown in Fig. 2. It proceeded much faster in solutions without $\mathrm{NaNO}_{2}$ because nitrite ions, having reducing properties, inhibit the photocatalytic process (Baran et al., 2003).

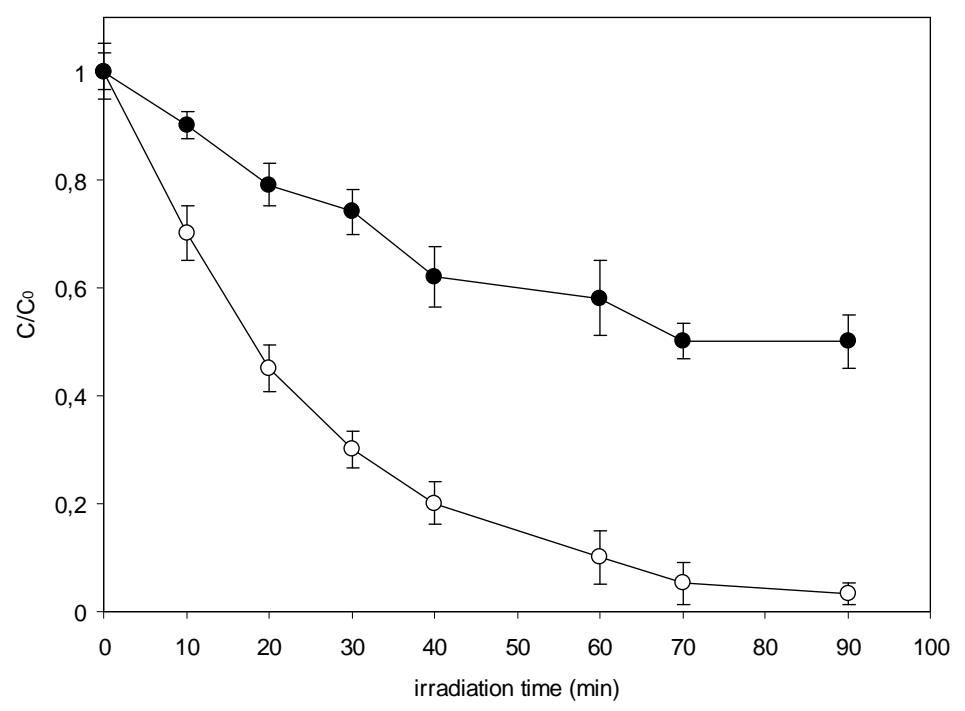

Figure 2: The dynamics of nicotine degradation $\left(C_{0} 0.1 \mathrm{mmol} / 1\right)$ during photocatalytic reactions: $(\bigcirc)$ with $\mathrm{TiO}_{2}(0.4 \mathrm{~g} / \mathrm{l}) ;(\bigcirc)$ with $\mathrm{TiO}_{2}(0.4 \mathrm{~g} / \mathrm{l})$ and $\mathrm{NaNO}_{2}(0.1 \mathrm{mmol} / \mathrm{l})$; error bars represent standard deviations from 3 replicates

In order to assess the possibility of nitrosamines formation during photocatalytic degradation of nicotine, the comparison of a mass spectrum of the initial nicotine solution (after adding the photocatalyst but before UV-a irradiation, Fig. 3a) and the spectra of nicotine products (after photocatalytic degradation) was carried out. 

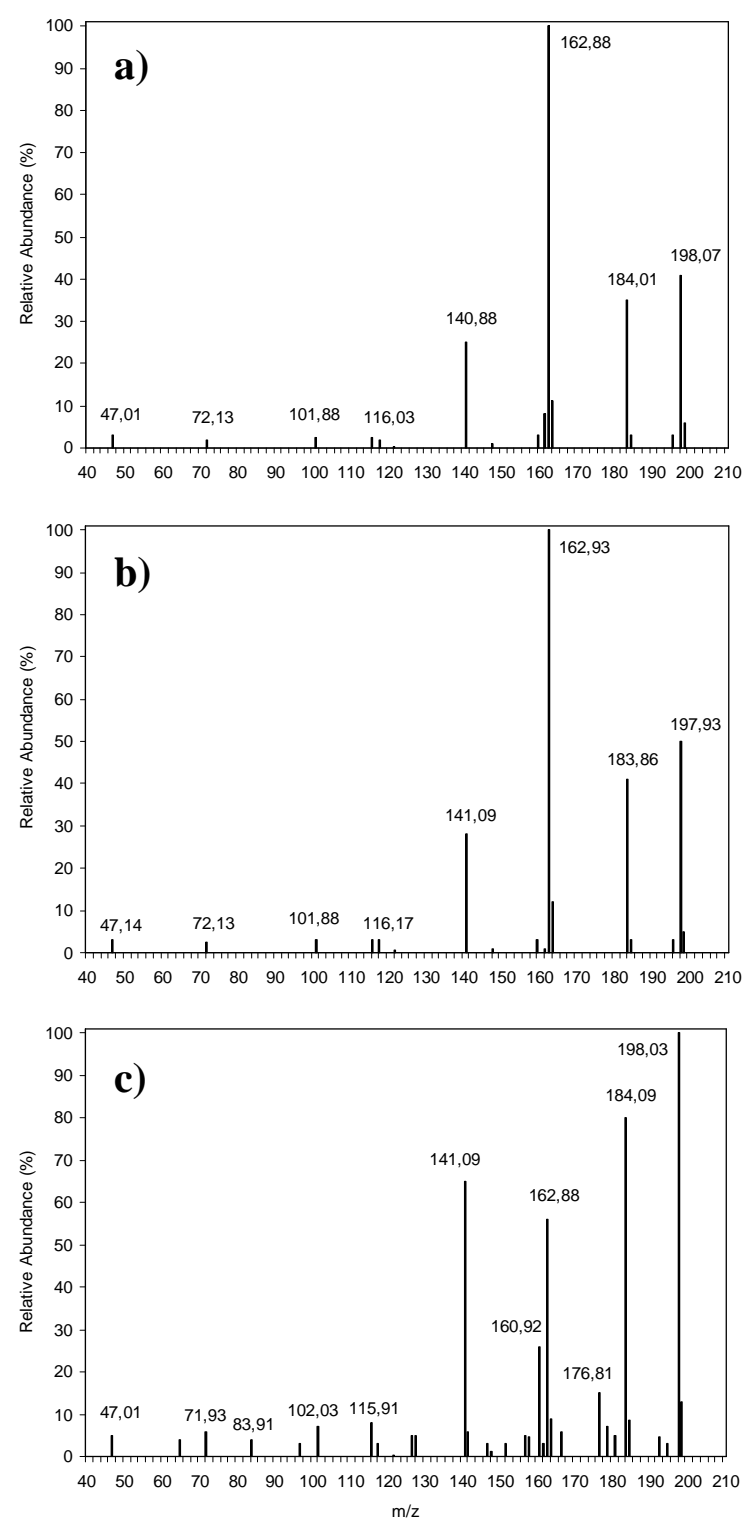

Figure 3: The MS/MS spectrum: (a) of the initial nicotine solution, (b) of sample containing products of photocatalytic degradation of nicotine, in the presence of $\mathrm{TiO}_{2}$ and $\mathrm{NO}_{2}$-ions, (c) of products of nicotine photodegradation in the presence of $\mathrm{TiO}_{2}$

It was stated that the compared spectra (Figs. 3a and $3 \mathrm{~b}$ ) do not show significant differences (Adamek et al., 2013). The reason for this is a low level of nicotine degradation. However, it should be noted that there are no peaks corresponding to the two investigated nitrosamines $(\mathrm{m} / \mathrm{z}$ 148 and 122) in reaction products. It may indicate that 4-(methylnitrosamino)-1-(3pyridyl)-1-butanone and N-nitrosonornicotine will not form during the photocatalytic degradation of nicotine in the presence of nitrite ions and possible other $\mathrm{NO}_{\mathrm{x}}$.

On the other hand, oxygen compounds of nitrogen may also be formed during the photocatalytic degradation of organic compounds containing nitrogen(s) in the molecules (Carp et al., 2004, Fujishima et al., 2008, Ohtani, 2010). In this case, the formation of nitrosamines is also possible 
but only at a higher level of nicotine conversion. Figure $3 \mathrm{c}$ shows a mass spectrum of nicotine solution UVairradiated for $60 \mathrm{~min}$ in the presence of $\mathrm{TiO}_{2}$ only. It was stated that after that time, nearly $90 \%$ of nicotine was degraded (Fig. 2).

The mass spectrum significantly differs from the initial spectrum of nicotine solution (Fig. 3a). These differences are likely the result of a high level of nicotine decomposition and they indicate that nicotine undergo transformation to other compounds. However, similarly as in the above-mentioned experiments, the investigated mass spectrum does not have peaks corresponding to nitrosamines. Therefore, there is no risk of their formation during photocatalytic process with $\mathrm{TiO}_{2}$.

\section{Conclusion}

Nicotine underwent degradation during photocatalytic process in the presence of $\mathrm{TiO}_{2}$. Identification of degradation products of nicotine, verified by LC-MS/MS analysis, confirmed that 4-(methylnitrosamino)-1(3-pyridyl)-1-butanone and N-nitrosonornicotine were not found in samples after their UVa-irradiation. Based on the results from the present study it can be concluded that photocatalytic process in the presence of $\mathrm{TiO}_{2}$ could be also used for the degradation of pollutants containing of nicotine.

\section{Acknowledgements}

This research was supported by the National Science Centre Grant funded by the Polish Government (UMO2011/03/D/NZ7/01684).

\section{References}

1. Adamek, E. Goniewicz, M. Baran,W. Sobczak, A. (2013) "The study on the photocatalytic degradation of nicotine", Proceedings of ECOpole, 7 187-192.

2. Baran, W. Makowski A. and Wardas, W. (2003) "The influence of inorganic ions on the photocatalytic degradation of orange II in aqueous solutions", Environment Protection Engineering, 6 75-85.

3. Baran, W. Sochacka, J. and Wardas, W. (2006) "Toxicity and biodegradability of sulfonamides and products of their photocatalytic degradation in aqueous solutions", Chemosphere, 65 1295-1299.

4. Bezak-Mazur, E. and Adamczyk, D. (2010) "Adsorption of mixture of two dyes on activated carbon", Proceedings of ECOpole, 4 307-312.

5. Carp, O. Huisman, C.L. and Reller, A. (2004) "Photoinduced reactivity of titanium dioxide", Progress in Solid State Chemistry, 32 33-177.

6. Fujishima, A. Zhang, X. and Tryk, D.A. (2008) " $^{\mathrm{TiO}_{2}}$ photocatalysis and related surface phenomena", Surface Science Reports, 63 515-582.

7. Hecht, S.S. Chen, C.B. Ornaf, R.M. Jacobs, E. Adams, J.D. and Hoffmann, D. (1978) "Reaction of nicotine and sodium nitrite: formation of nitrosamines and fragmentation of the pyrrolidine ring", Journal of Organic Chemistry, 43 72-76.

8. Hecht, S.S. Carmella, S.G. Foiles, P.G. Murphy, S.E. and L.A. Peterson, (1993) "Tobacco-specific nitrosamine adducts: studies in laboratory animals and humans", Environmental Health Perspectives, 99 5763.

9. Ohtani, B. (2010) "Photocatalysis A to Z-What we know and what we do not know in a scientific sense", Journal of Photochemistry and Photobiology C: Photochemistry Reviews, 11 157-178.

10. Sleiman, M. Gundel, L.A. Pankow, J.F. Jacob III, P. Singer, B.C. and Destaillats, H. (2010) "Formation of carcinogens indoors by surface-mediated reactions of nicotine with nitrous acid, leading to potential thirdhand smoke hazards", Proceedings of the National Academy of Sciences of the United States of America, 107 6576-6581. 